\title{
Reflecting Backward, Moving Forward
}

\author{
Darshana Shah, $\mathrm{PhD}^{1}$
}

\section{KEYWORDS}

Author affiliations are

listed at the end of this article.

Correspondence to: Darshana Shah, PhD Marshall Unviersity Joan C. Edwards School of Medicine shah@marshall.edu

Reflecting Backward, Moving Forward

As the Marshall Journal of Medicine enters its sixth year of publication, there is a lot to look forward to. Before looking ahead, however, I would like to offer a word of thanks to our readers, our contributors, and our editorial board for their support of the journal and its mission: to improve and expand knowledge of rural health care for practitioners, educators, researchers, and policymakers in Appalachia and beyond. Launching a new journal is no small feat, and we have achieved a good measure of success with 19 on-time issues and consistent, high-quality content from outstanding contributors.

\section{Serving as editor-in-chief of the Marshall Journal} of Medicine (MJM, the Journal) has been one of the highlights of my professional career. I am writing to give you an update on the progress that the Marshall Journal of Medicine has made during the last five years.

MJM has published a total of 200 peer-reviewed articles in diverse submission categories with high-quality content received from local, regional, national, and international contributors. In five years of publication, the Journal global readership map has expanded and includes 198 countries with greater than 72,000 downloads since its inception. The Journal is indexed in Google Scholar, CLOCKSS, Portico, OCLC (WorldCat), ProQuest, Serial Solutions/ Summon, EBSCO Discover, and is a member of the Directory of Open Access Journal (DOAJ) and the Committee on Publication Ethics (COPE).

In order to further improve the status of MJM, we have introduced several new measures. The first includes measuring impact beyond Google Scholar and Crossref through our partnership with PlumX, a service that helps provide a deeper understanding of how your published research is being used. PlumX gathers data from any online forum where published research is being discussed, be it social media, news outlets, or even policy documents and patents, and displays it as a nifty and colorful badge on the article page on the MJM website. Each published article now shows the PlumX metrics widgets and download counts, which are helpful tools for authors to see their impact. PlumX metrics give better insight into user engagement with the publication by tracking factors such as the number of downloads and mentions of research in news articles or tweets. 
The Journal also now offers Native Streaming, a new feature supported by Digital Commons. Native Streaming allows authors to upload multimedia files as the primary file during submission; it includes usage metrics, supports closed-captioning, and provides unlimited storage space. Additionally, readers will be able to view content without having to download it. Video abstracts, an additional feature, increase the visibility of our authors and their work. Through streaming video media, authors can now expand the reach of published articles and provide a new, enhanced user experience for the Journal's global audience.

An additional feature offered by MJM is the Author Dashboard, a personalized reporting tool for authors with works published in MJM. An author can easily access the dashboard to view up-to-date download information for every work published, as well as global insights into the sources of readership. There are also options to share data with others and export statistics for use in external applications.

Through a mix of technology, improved procedures, and the assistance and commitment of our peerreview team, board members, and referees, we are delighted to announce that the median time for receiving the initial decision from MJM is now reliably under 35 days and still improving. Consequently, the median time for a paper to be accepted following submission is now just under four months. Importantly, this has been achieved without compromising the strict quality standards for peer review that the journal is well-known for.

In summary, the status of the Marshall Journal of Medicine is excellent and the outlook is positive. I would like to sincerely thank all that have made this possible. My special thanks go out to the entire MJM Editorial Board and the many anonymous reviewers who ensure that submitted papers are assessed carefully, fairly, and in a timely manner. Especially during this surreal time, we wish health and safety to all of our readers. The editorial staff and I appreciate your dedication to the Marshall Journal of Medicine. 